Www.jmscr.igmpublication.org GM Publication
Journal Of Medical Science And Clinical Research

An Official Publication of IGM Publication

\title{
MR Evaluation of Isolated Fallopian Tubal Torsion, Rare Cause of Lower Abdominal Pain in Perimenopausal Age - Report of Two Cases and Mini Review
}

Authors

\section{Dr. Ankit Agrawal ${ }^{1}$, Dr. Praveen Kumar $\mathbf{P}^{2}$}

${ }^{1} \mathrm{II}^{\text {nd }}$ year postgraduate Student, Department of Radiology, Navodaya Medical College, Raichur, Karnataka (State), India - 584103

${ }^{2}$ Associate Professor, Department of Radiology, Navodaya Medical College,

Raichur, Karnataka (State), India - 584103

Corresponding Author

Dr. Ankit Agrawal

Postgraduate Student, Department of Radiology, Navodaya Medical College, Mantralayam Road

Post: Raichur, Karnataka (State), India - 584103 Mobile Number: +918861233006

Email: ankitag2008@yahoo.com

\section{Abstract:}

Introduction: Isolated fallopian tubal torsion is a rare entity causing lower abdominal pain, occurring predominantly in the reproductive age group and common on right side. Certain risk factors like hydro/hematosalpinx, previous tubal ligation, abnormal mesosalpinx and hypermotility of tube predispose to tubal torsion. Most cases are usually undiagnosed pre operatively because of non-specific symptoms, physical findings and imaging features.

Case Reports: Here we are presenting two women of perimenopausal age presenting with fallopian tubal torsion with previous history of tubal sterilization, which were diagnosed preoperatively by MR imaging.

Conclusions: Isolated fallopian tubal torsion should be considered in the differential diagnosis for acute lower abdominal/pelvic pain in women of any age group with particular risk factors. Sometimes ultrasound may not be able to diagnose this condition preoperatively, in such cases MRI imaging plays an important role and help in prompt intervention to avoid further catastrophic complications.

Key words: Fallopian tube, Torsion, Perimenopausal age, Magnetic Resonance Imaging

\section{Introduction}

Isolated fallopian tubal torsion is a rare event presenting with acute lower abdominal or pelvic pain. It is common in the reproductive age group particularly on the right side. Ultrasonography is used for initial evaluation in such cases, while CT
(Computed tomography) and MR (Magnetic Resonance) imaging used for confirmation of diagnosis in some instances. It is usually diagnosed peroperatively during laparoscopy or laparotomy. Only less than $20 \%$ of reported cases were diagnosed preoperatively by imaging, but a 
high index of suspicion is critical for making the correct diagnosis. Early diagnosis and treatment will help avoiding catastrophic complications like tubal gangrene and thereby preserve fertility. Here we present two cases of isolated fallopian tubal torsion which were later confirmed by surgical findings.

Case 1: A 51 year old perimenopausal woman presented with acute onset of severe pain and discomfort in the left lower abdominal and pelvic region when she was about to board a flight. There was no history of fever, cough, breathlessness, and pervaginal discharge. On physical examination pulse rate and blood pressure were normal. Tenderness was noted in the left iliac fossa and pelvic region. No guarding or increased bowel sounds were present. Pervaginal examination showed a tender mass in the left adnexal region. The patient had tubal sterilization 15 years back.

Ultrasound examination done in outside institution showed a cystic area in the left adnexa, but could not visualize left ovary. T2 weighted MRI images of the pelvis showed an elongated tubular cystic structure in the left adnexa, which was seen separately from the left ovary and uterus. Multiple blood / fluid levels were noted within the tubular cystic structure at the base associated with adjacent fat stranding (Fig I and II). Contrast enhanced CT pelvis also showed an elongated tubular cystic structure with thick peripheral wall enhancement in the left adnexa and sterilization band at its base(Fig III). Adjacent significant soft tissue fat stranding and minimal free fluid in the pelvis were also seen. The right ovary was visualized in right adnexa and pouch of Douglas and showed few small follicles.

Based on history, clinical and imaging findings, a final diagnosis of left fallopian tubal torsion secondary to tubal sterilization was made. Patient then underwent laparotomy which revealed a large twisted hydrosalpinx in the left adnexa, appearing dusky blue in color with the tubal band at its base. Both ovaries were seen separately from this lesion. The twisted segment of left fallopian tube was excised and sent for histopathological examination. Histopathological examination of the twisted hydrosalpinx showed inflammatory changes with areas of necrosis and hemorrhage within the thickened wall.

Case 2: A 44 year old woman presented with persistent nagging pain in the right iliac fossa for 3 months. There was no history of fever, vomiting or pervaginal bleeding. She had history of tubectomy 12 years back. She did not have any gynecological complaints in the past. Transabdominal ultrasound done showed only normal uterus and ovaries with minimal free fluid in the pelvis. MR images showed right hydrosalpinx which was oriented vertically with twisted appearance at the base and seen separately from the right ovary (Fig IV and V). Left ovary and uterus were normal (Fig V). Based on these findings a preoperative diagnosis of isolated right fallopian tubal torsion was done and later patient underwent laparotomy. On laparotomy, a twisted right fallopian tube without gangrene was confirmed.

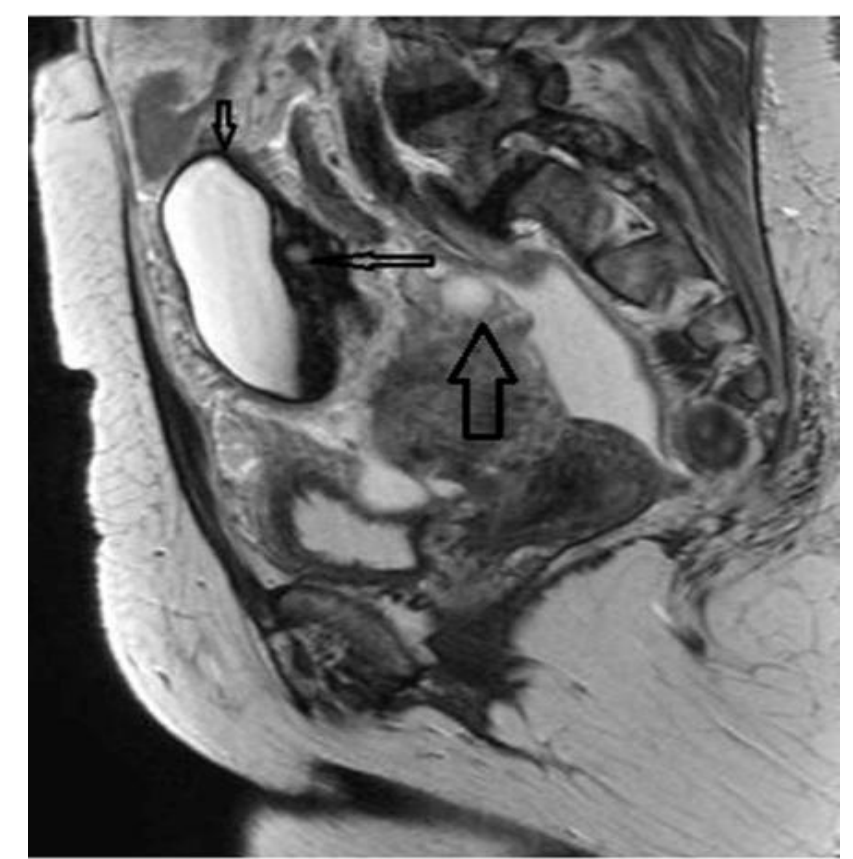

Fig I: 51 year old woman with isolated left fallopian tubal torsion. Sagittal T2 Wt image of the pelvis shows an elongated cystic structure within the left adnexa (short arrow) seen separately from the left ovary (thick arrow), with 


\section{JMSCR Vol||3||Issue||9||Page 7689-7693||September}

blood fluid levels at the site of torsion (long arrow)

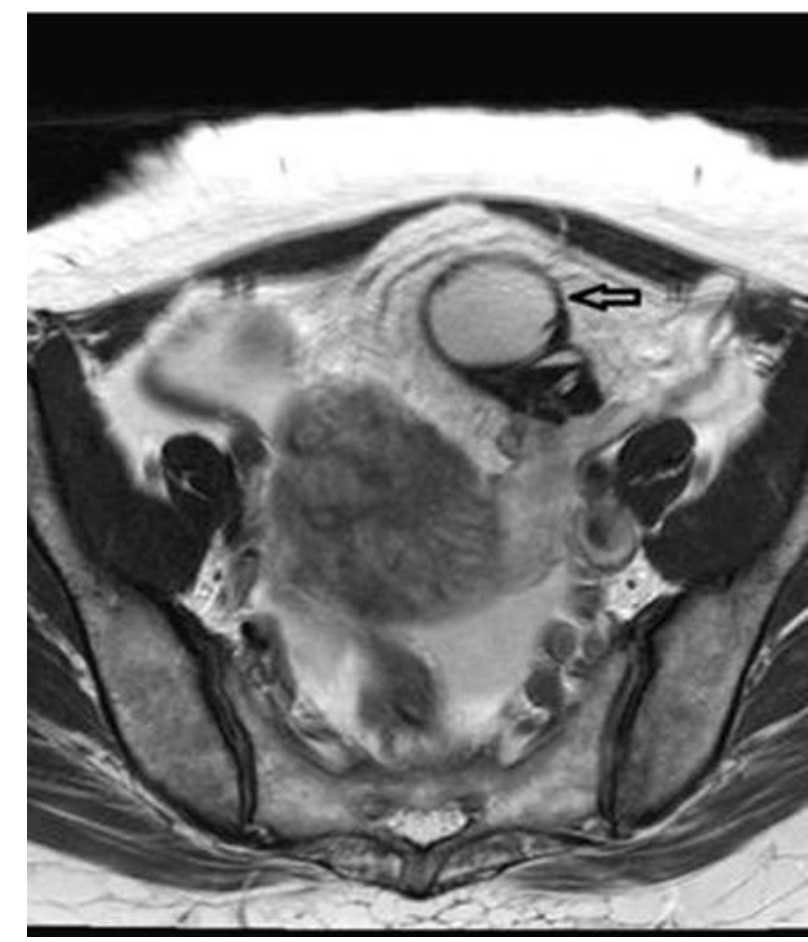

Fig II: 51 year old woman with isolated left fallopian tubal torsion. Axial T2 Wt image of the pelvis shows a cystic structure within the left adnexa (short arrow) with blood fluid levels at the site of torsion

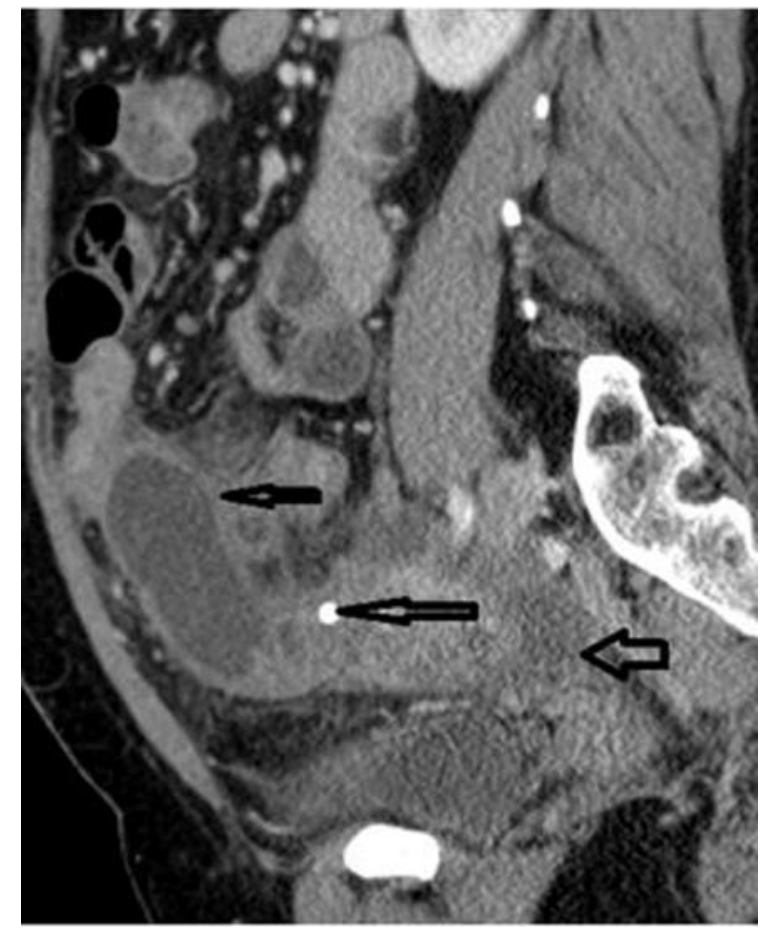

Fig III: 51 year old woman with isolated left fallopian tubal torsion. Contrast enhanced sagittal CT scan of the pelvis shows an elongated tubular cystic structure within the left adnexa (short arrow). Sterilization clip is noted postero-inferior to the cystic structure (long arrow). Minimal fluid is also noted within the pouch of Douglas (thick arrow)

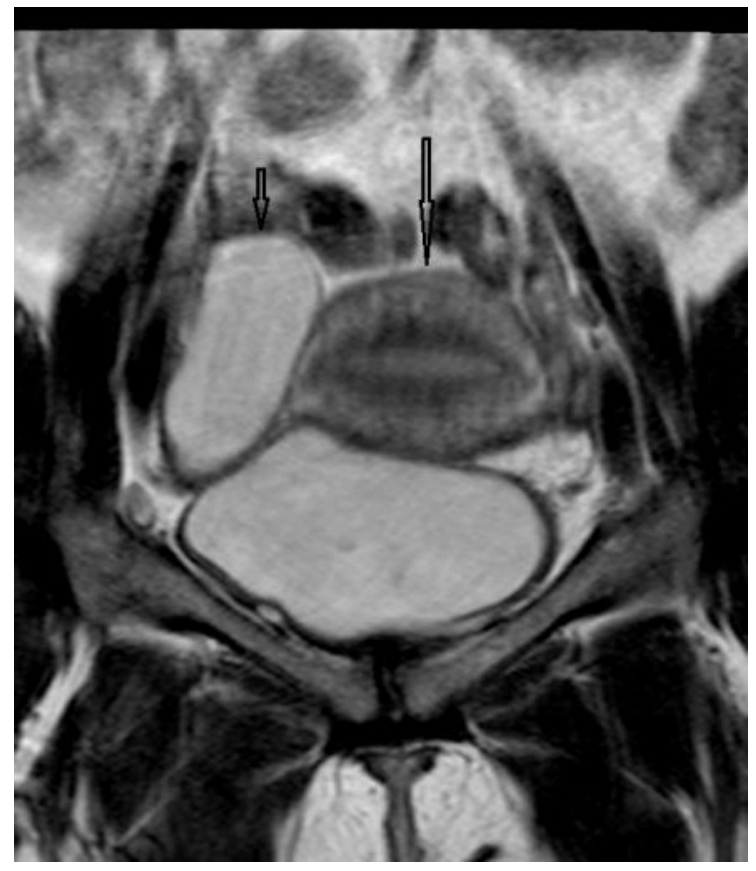

Fig IV: 44 year old woman with isolated right fallopian tubal torsion. Coronal T2 image shows an elongated tubular structure in the right adnexa (short arrow) which is oriented vertically lateral to the uterus (long arrow)

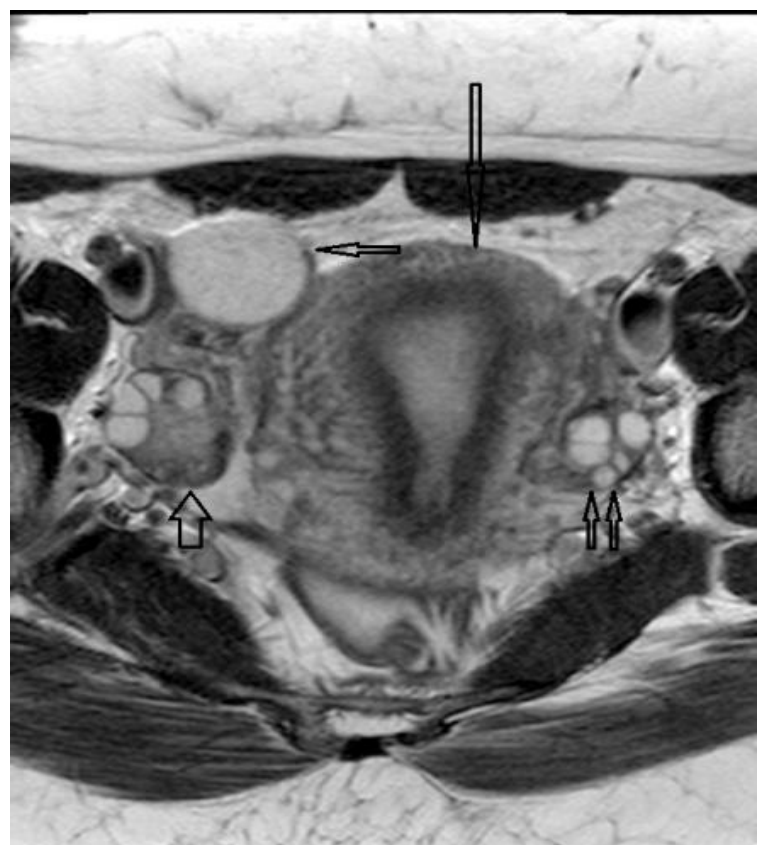

Fig V: 44 year old woman with isolated right fallopian tubal torsion. Axial T2 image shows a 
tubular structure in the right adnexa (short arrow) situated lateral to the uterus (long arrow) and separately seen from the right ovary (thick arrow). Left ovary is seen in left adnexa(double arrow)

\section{Discussion}

Adnexal torsion (ovarian and tubal) is relatively uncommon and should be considered in the differential diagnosis of acute abdominal or pelvic pain in women, with the ovarian torsion being the common entity. Isolated tubal torsion is very uncommon with reported incidence of 1 in $15,00,000$ women and was described originally by Bland Sutton in $1890^{(1,2)}$.

The exact cause for tubal torsion is not known. Some of the risk factors or aetiologies which may predispose to torsion include long mesosalpinx, venous congestion in the mesosalpinx, hydro/hematosalpinx, hypermobility of the tube, tubal spasm, tubal ligation, trauma / surgery, pelvic inflammatory disease, gravid uterus, and previous hysterectomy ${ }^{(3)}$.

Though torsion can occur in a normal fallopian tube, disruption of anatomic relations as happens after tubal sterilization and hysterectomy may enhance torsion. Moreover, sterilization procedures have been associated with hydrosalpinx formation, which may act as an additional weight, resulting in torsion ${ }^{(4)}$.

Right side tubal torsion is more common than on the left, because of the presence of sigmoid colon on the left (preventing tubal torsion), slow venous flow on right side and because right lower quadrant pain is more often surgically explored ${ }^{(5)}$. Left sided tubal torsion usually goes unrecognized, resulting in further complications.

The condition is uncommon before menarche and in perimenopausal women ${ }^{(6)}$, because most of the risk factors operate in the adolescents and ovulating women. Patients usually present with acute onset of lower abdominal and pelvic pain, which may be severe and intermittent or recurrent. The pain may radiate to the flank or thigh. The recurrent pain may indicate intermittent noninfarctive torsion, but acute severe pain is a sign of torsion with impeding infarction and gangrene. Other symptoms with which patients may present include nausea, vomiting, pervaginal bleeding, and bladder / bowel complaints. Blood investigations will be normal in most instances.

Cross sectional imaging methods like ultrasound, CT and MRI can be used for the diagnosis of tubal torsion. On ultrasound, an elongated tubular cystic structure with hyperechoic wall, folded configuration can be seen in the adnexa, which is seen separately from the ipsilateral ovary and uterus.

The tubular structure will be seen tapering towards the uterine cornu resulting in sonographic 'Whirlpool sign' (7). Doppler shows either high resistance or absent flow within the periphery of the cystic structure ${ }^{(8)}$.MR imaging findings include presence of elongated tubular adnexal mass, dilated tube $(>15 \mathrm{mms})$, thickened and enhancing walland beaking at the site of torsion. CT images show high attenuation contents suggestive of hemorrhage within the tubal lumen (HU > 50). Secondary signs include free fluid in the pelvis, peritubal inflammatory changes, local ileus and broad ligament thickening $(9,10)$. Depending upon the underlying aetiology, we can identify hydro or hematosalpinx, venous congestion within mesosalpinx, tubal bands secondary to sterilization procedures and gravid uterus.

In most instances, preoperative diagnosis of isolated tubal torsion based on imaging findings alone is not possible, because of nonspecific imaging findings. So, it should be correlated with history, presenting symptoms and physical findings.

Differential diagnoses for tubal torsion include torsion of ovarian cyst or subserosal fibroid, rupture of ovarian cyst or follicle, pelvic inflammatory disease, ectopic pregnancy, appendicitis, diverticulitis, intestinal obstruction, and urolithiasis. Complications of tubal torsion include tubal necrosis and gangrene, resulting in peritonitis and damage to the ipsilateral ovary. 
The condition can be treated either by detorsion, salpingotomy or salpingectomy, based on the stage of diagnosis and presence of complications. In earlier days, it was treated by laparotomy, but with the advent of lesser invasive techniques the condition can now be diagnosed and treated with laparoscopy.

Both the cases were unusual, in that they are seen in perimenopausal women and the first case was on left side.

\section{Conclusions}

Isolated fallopian tubal torsion is a rare cause of acute lower abdominal or pelvic pain in women. It is more common on the right side and in reproductive age group. Preoperative diagnosis of tubal torsion is difficult in most instances, because of overlapping clinical and nonspecific imaging findings. Tubal torsion should be considered in the differential diagnosis of acute abdominal or pelvic pain even in perimenopausal age group with risk factors as mentioned above. MR imaging plays an important role in few cases and helps in prompt intervention and reduces the morbidity and mortality from this condition.

\section{References}

1. Raziel A, Mordechai E, Friedler S, Schachter M, Pansky M, Ron-El R. Isolated recurrent torsion of the fallopian tube: case report. Hum Reprod 1999; 14:3000-3001

2. Ferrera PC, Kass LE, Verdile VP. Torsion of the fallopian tube. Am J Emerg Med 1995; 13:312-314.

3. Shukla R. Isolated torsion of the hydrosalpinx: A rare presentation. BJR, 77(2004), 784-786.

4. Krissi H, Orvieto R, Dicker D, Dekel A, Ben Rafael Z. Torsion of a fallopian tube following Pomeroy tubal ligation: a rare case report and review of the literature. EurJObstetGynecolReprod Biol. 1997; 72:107-109.
5. Richards, H.M, Parsons, R.B, Broadman, K.F. et al. (1998) Torsion of the fallopian tube: progression of sonographic features. J. Clin. Ultrasound, 27, 374-376.

6. Bharathi A, MangalaGowri. Torsion of fallopian tube and hematosalpinx in perimenopausal women-A case report. Journal of Clinical and Diagnostic Research. 2013 April, Vol-7(4): 731-733

7. BoopathyVijayaraghavan S, SathiyaSenthil. Isolated torsion of the fallopian tube - The sonographic Whirlpool Sign. J Ultrasound Med 2009; 28:657-662.

8. Elchalal U, Caspi B, Schachter M, Borenstein R. Isolated tubal torsion: clinical and ultrasonographic correlation. J Ultrasound Med 1993; 12:115-7.

9. Ghossain MA, Buy JN, Bazot M, et al. CT in adnexal torsion with emphasis on tubal findings: correlation with US. J Comput Assist Tomogr 1994; 18:619 -625.

10. Megan Gross, Sylvie L. Blumstein, Lawrence C. Chow. Isolated fallopian tube torsion: A rare twist on a common theme. AJR 2005; 185:1590-1592

Financial conflicts: None 